\title{
Survey and revision of leaf miners to some plants from different localities of Iraq
}

\author{
Hanaa H Al-Saffar $1{ }^{1}{ }^{*}$ Razzaq Shalan Augul ${ }^{1}$ and Zainab Abid Aun Ali ${ }^{2}$ \\ ${ }^{1}$ Iraq Natural History Research Center and Museum/ University of Baghdad. \\ ${ }^{2}$ College of Science for Women/ University of Baghdad.
}

GSC Biological and Pharmaceutical Sciences, 2021, 17(03), 124-136

Publication history: Received on 11 November 2021; revised on 12 December 2021; accepted on 14 December 2021

Article DOI: https://doi.org/10.30574/gscbps.2021.17.3.0358

\begin{abstract}
This investigation showed (31) species belonging to (15) genera under (five) families and two orders. The leafminers Dipter families (Agromozidae, Anthomyiidae, Drosophilidae), Agromyzid flies is the highest level of investigated many host plants, but other families have lowest host plants. The synonyms of species were provided from GBIF scarlet's. The date and localities of sampling collection were recorded.
\end{abstract}

Keywords: Leaf miners; Agromyzidae; Anthomyiidae; Drosophilidae; Lepidoptera; Iraq

\section{Introduction}

The leaf miner, showed any of insect larvae which habitats and feed in leaf plants. The miners include caterpillars of some lepidopterans (Order Lepidoptera), sawfly larvae (Order Hymenoptera), beetle and weevil grubs or larvae (order Coleoptera), and maggots (larvae) of true flies (order Diptera [1]

Most leaf-miner of order Diptera is Family Agromyzidae made burrows or tunnels in leaves of host plants, some of them are stem borer with galls maker [2]. This family is widely distributed at the world with loss species southern part of hemisphere than in hot areas of Nearctis and Palaearctic regions then it had many studies in deferent localities $\mathrm{f}$ the world $[3,4]$.

Agromyzidae consist of more than 2,500 species round the world, its member's small with wing length2-3mm in maximum about $6.5 \mathrm{~mm}$. The adults can be diagnosed with characters; head sclerotizes and the upper part of frons lightly sclerotized and loss seta, but the lower and dorsal of head with more heavily sclerotized with setulae and pubescent, frontal vita sometimes made patch and tuff in different colors compare with the rest of head. The frons with 1-7 frontal bristles, with presence of vibrissae. Compound eyes ovate in shape, small usually but in some species circular and large. Wings usually hyaline, often with marks (dark spots and shaped) of tropical spcies. Costal vein has break on the apex of subcost; cup cell small; $1^{\text {st }}$ anal vein not reached the wing margen. Abdomen has pach andcolor spot on tergites in male using to differ from species, female abdomen fused tergal ( $\mathrm{T}$ 6-8) which has two spiracles located between (T5 and genital segment) while the seventh one forming an oviscape. $[2,5,6,7,8,9]$.

Family Anthomyiidae is one of the most important flies which distributes at all the zoogeographical region. They found with deferent conditions from vegetables, dead animals and most of them phytophagus caused injuries and damaged plants, such genera Delia and Pegomya; (Pegomya hyoscami species group). Some species had economic importance which infested cultivated crops [10].

\footnotetext{
* Corresponding author Hanaa H Al-Saffar

Iraq Natural History Research Center and Museum/ University of Baghdad.

Copyright ( $(2021$ Author(s) retain the copyright of this article. This article is published under the terms of the Creative Commons Attribution Liscense 4.0.
} 
The Spinach leafminer Pegomya hyoscyami small fly, female inserted ovipositor and laid eggs on leaf surface and maggot made tunnel on it. Larvae whit maggot caused blotches, then pupal stage and adults emerge in spring from soil. [11].

The family Drosophilidae is a cosmopolitan of acalyptrate consists of more than 3,950 species [12]. The important genus is Scaptomyza Hardy, a lot of species belong to it as leaf miners. Scaptomyza flava is an alienate species of European origin [13]

The most important lepidopteran leafminers (Tomota Leaf miner and Citrus leaf miner). Which economically importance. Tuta absoluta is phytophagus insect attacking Solanaceae family plants [14].

\title{
2. Material and methods
}

Many infested leaf of plants were collected from different Localities of Iraq, (25-40) leaves per each plants) during 2020 year. The collecting leaves were brought to the department of Entomology and Invertebrates at Iraq Natural History Research Center and Museum, then kept in Petri Dishes under room temperature. The dishes were labeled and the places and date of collecting were recorded. After 2-3 weeks the insect left leaves as adults.

The plants and insects of orders Diptera and Lepidoptera were diagnosed by authors and used taxonomical keys such as: $[2,8,10,11]$ The specimens were storage at department of Entomology and invertebrate.

\section{Results and discussion}

This investigation showed (31) species belonging to (15) genera under (5) families with two orders (Diptera and Lepidoptera) as fallow:

\author{
Order, Diptera \\ Family, Agromyzidae \\ Subfamily, Agromyzinae \\ Genus, Agromyza Fallén, 1810 \\ Synonyms: Adromyza Meigen, 1830 \\ Calyptomyza Hardy, 1850 \\ Mesonevra Lioy, 1864 \\ Stomacrypolus Enderlein, 1936
}

\subsection{Agromyza albipennis Meigen, 1830}

Synonyms: Agromyza albohyalinata Zetterstedt, 1848

Agromyza dubitata Malloch, 1913

Agromyza fennica Griffiths, 1963

Common name: black wheat leaf miner

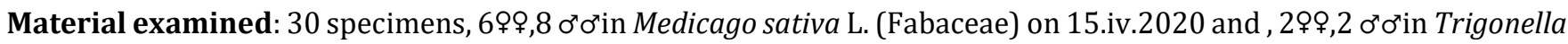
foenum-graecum L. (Fabaceae)on 21.ii.2020 from Baghdad; , 3ㅇ,3 ơ ơin Hordeum vulgare on 10.iv.2020 from Al-Najaf; , 4ㅇ,2 ơ o'in Meloletis indica on 10.iv.2020.from Kerbala.

Distribution: Iraq[15]; Denmark, Finland, Norway, Sweden, Germany[16] Austria, Belarus, Corsica, Czech Republic, Estonia, European Turkey, Finland, Hungary, Italian mainland, Latvia, Lithuania, Poland, Slovakia, Spanish mainland and Switzerland; Also recorded in Canada, Japan and China [17]; Netherlands, Luxembourg , Belgium[18]; Turkey [19] Portugal [20].

\subsection{Agromyza megalopsis Hering, 1933}

Material examined: 4specimens, 2९o, $2 \sigma^{\top} \sigma^{\prime}$ in Triticum aestivum L. (Poaceae) on 30.iii. 2020 from Baghdad. Distribution: Iraq [21]; in Europe, it's distributed from Poland to Spain and from France to Crete [22]; Bulgaria, Corfu, Crete and Croatia [23]; Former Yugoslavia; Egypt, Cyprus, Saudi Arabia, Turkey, Uzbekistan, Morocco [24].

\subsection{Agromyza nana Meigen, 1830}

Synonyms: Agromyza anthracipes (Rondani, 1875)

Agromyza brevinervis (Rondani, 1875)

Agromyza medicaginis Robineau-Desvoidy, 1851 
Agromyza trifolii Kaltenbach, 1872

Domomyza anthracipes Rondani, 1875

Domomyza brevinervis Rondani, 1875

Material examined: 20 specimens, 6\%९, 6 ơ $^{\top}$ in Melilotus indeca (L.) All. (Fabaceae) at 13.ii.2020 from Kerbala; 6\%९, 2 ơ' ơin Medicago sativa L. (Fabaceae) on 4.ii.2020. From Baghdad.

Distribution: Iraq [25] Palearctic Region [26]; Turkey [19] Portugal [20].

\subsection{Agromyza nigrescens Hendel, 1920}

Synonyms: Agromyza heringi Meijere, 1925

Agromyza japonica Tsujita, 1951

Agromyza microchaeta Hendel, 1920

Agromyza oycoviensis Beiger, 1960

Common name: Geranium leaf miner

Material examined: 4specimens, 3ㅇ, $1 \sigma^{\prime}$ in Geranium dissectum L. (Geraniaceae) on 3.iii.2020 from Baghdad.

Distribution: Iraq [15]; Denmark, Finland, Germany, Norway [16]; Turkey [19]; Netherlands, Greece, Luxembourg, UK, Switzerland, Spain, Japan, Croatia, Cyprus, Colombia, Italy, Sweden, Austria, Switzerland, Estonia [27].

Genus, Melanagromyza Hendel, 1920

Synonym: Limnoagromyza Malloch, 1921

\subsection{Melanagromyza azawii Spencer, 1973}

Material examined: This species previously collected from stem of Vicia faba L. (Fabaceae) and Sesamum indicum L. (Pedaliaceae) on July 1979 [15].

Distribution: Iraq and Greece [15]; Palaearctic Region [29]; India and Somalia [30]; Secretariat, 2021 b).

Genus, Phytoliriomyza Hendel, 1931

Synonyms: Lemurimyza Spencer, 1965

Nesomyza Spencer, 1973

Pteridomyza Nowakowski, 1962

Xyraeomyia Frick, 1952

\subsection{Phytoliriomyza oasis (Becker, 1907)}

Synonym: Agromyza oasis Becker, 1907

Material examined: We cannot collect this species but previously recorded on March in Mousl[15].

Distribution: Iraq [15]; Kyrgyzstan, Lithuania, Morocco, Poland, Spain, Sweden, Turkey and Uzbekistan [22]; Turkey [19]; Portugal [20]; Norway and Greece [27].

Genus: Ophiomyia Braznikov, 1897

Synonyms: Aulomyza Enderlein, 1936

Carinagromyza Sasakawa, 1954

Siphonomyza Enderlein, 1936

Siridomyza Enderlein, 1936

Solenomyza Enderlein, 1936

Stiropomyza Enderlein, 1936

Stirops Enderlein, 1936

Tylomyza Hendel, 1931

\subsection{Ophiomyia phaseoli (Tryon, 1951)}

Synonyms: Agromyza destructor Malloch, 1916

Agromyza fabae Tryon, 1897

Agromyza fabalis Jack, 1953

Agromyza phaseoli Coquillett, 1899

Ophiomyia gangetica Garg, 1971

Ophiomyia sanctuarii Singh \& Ipe, 1973

Oscinis fabae Tryon, 1897

Oscinis phaseoli Tryon, 1895 
Common name: Bean fly; snap bean fly.

Material examined: 10 specimens, (5ㅇ,5 $\sigma^{\circ} \sigma^{x}$ ) in Vigna unguiculata (L.) Walp. (Fabaceae) on $5 . v .2020$ from AbuGhraib/Baghdad.

Distribution: Iraq [15]; (Spencer, 1981); Afrotropical, Australian, Oriental and Palaearctic Regions [29]; Turkey [19]; Bangladesh [31].

\section{Subfamily, Phytomyzinae \\ Genus, Calycomyza Hendel, 1931}

\subsection{Calycomyza humeralis (Roser, 1840)}

Synonyms: Agromyza atripes Brischke, 1881

Agromyza bellidis Kaltenbach, 1873

Agromyza humeralis von Roser, 1840

Calycomyza bellidis (Kaltenbach, 1873)

Calycomyza humerella (von Roser, 1840)

Material examined: 4 specimens, $39 \%$ and $10^{\prime}$ in Tripolium pannonicum subsp. Tripolium (L.) Greuter (Asteraceae) on 4.iii.2020 from Tarmiyah/ Baghdad.

Distribution: Iraq 15]; Turkey [19]; Argentina, Germany, Bulgaria, Norway, Switzerland, Netherlands, UK, South Africa, Zimbabwe, Ethiopia, Australia, Canada, USA [30].

Genus, Cerodontha Rondani, 1861

Synonyms: Cerodonta Hendel, 1910

Cerodonta Hering, 1926

\subsection{Cerodontha denticornis (Panzer, 1806)}

Synonyms: Agromyza acuticornis Meigen, 183

Agromyza confinis Meigen, 1830

Agromyza nigritarsis Meigen, 1830

Agromyza tarsella Zetterstedt, 1848

Ceratomyza nigriventris Strobl, 1900

Ceratomyza nigroscutellata Strobl, 1900

Ceratomyza semivittata Strobl, 1909

Cerodontha acuticornis (Meigen, 1830)

Cerodontha confinis (Meigen, 1830)

Cerodontha ghooma Singh \& Ipe, 1973

Cerodontha lacustris Garg, 1971

Cerodontha meigenii (Fallén, 1823)

Cerodontha narkandae Singh \& Ipe, 1973

Cerodontha nigriscutellata (Strobl, 1900)

Cerodontha nigritarsis (Meigen, 1830)

Cerodontha nigriventris (Strobl, 1900)

Cerodontha semivittata (Strobl, 1909)

Cerodontha tarsella (Zetterstedt, 1848)

Cerodontha testae Singh \& Ipe, 19731

Chlorops denticornis Panzer, 1806

Chlorops meigeni $\mathrm{Pa} 1984$

Chlorops meigenii Fallen, 1823

Material examined: 1 specimen in Alopecurus partensis L. (Poaceae)on 15.iii.2020 from Sulaymaniyah city.

Distribution: Iraq[15]; Iran[32]; Turkey[19]; Albania, Andorra, Austria, Belgium, Bulgaria, Czech, Dalmatia, Denmark, Estonia, Finland, France, Germany, UK, Greece, Hungary, Ireland, Italy, Latvia, Lithuania, Maltese Islands, Montenegro, Netherlands, Norway, Poland, Portugal, Romania, Slovakia, Spain,, Sweden, Switzerland, Ukraine, North Africa, Central and East Asia[20].

\subsection{Cerodontha luctuosa (Meigen, 1830)}

Synonyms: Agromyza luctuosa Meigen, 1830

Cerodontha effusi (Karl, 1926)

Dizygomyza effusi Karl, 1926 
Material examined: 2 ㅇ, specimens in Juncus effuses L. (Juncaceae) on 17.iii.2020.

Distribution: Iraq[15]; Turkey[19]; Albania, Austria, Belgium, Belarus, Czech, Denmark, Finland, France, Germany, Greece, Hungary, Ireland, Italy, Latvia, Lithuania, Netherlands, Norway, Poland, Portugal, Russia, Slovakia, Spain, Sweden, Switzerland, former Yugoslavia, Israel, Tunisia, China, Japan, Uzbekistan, Canada and United States[20].

\section{Genus, Chromatomyia Hardy, 1849}

\subsection{Chromatomyia horticola Goureau, 1851}

Synonyms: Chromatomyia bidensivora (Séguy, 1951)

Chromatomyia cucumidis (Macquart, 1854)

Chromatomyia fediae (Kaltenbach, 1860)

Chromatomyia lactucae (Vimmer, 1928)

Chromatomyia linariae (Kaltenbach, 1862)

Chromatomyia meliloti (Brischke, 1882)

Chromatomyia nainiensis (Garg, 1971)

Chromatomyia pisi (Kaltenbach, 1864)

Chromatomyia subaffinis (Malloch, 1914)

Chromatomyia tropaeoli (Dufour, 1857)

Napomyza lactucae Vimmer, 1928

Phytomyza bidensivora Séguy, 1951

Phytomyza cucumidis Macquart, 1854

Phytomyza fediae Kaltenbach, 1860

Phytomyza horticola Goureau, 1851

Phytomyza linariae Kaltenbach, 1862

Phytomyza meliloti Brischke, 1882

Phytomyza nainiensis Garg, 1971

Phytomyza pisi Kaltenbach, 1864

Phytomyza subaffinis Malloch, 1914

Phytomyza tropaeoli Dufour, 1857

Common name: Pea leaf miner

Material examined: 45 specimens; 69\%, 6 ơ ơin Lepidium darba L. (Brassicaceae) on 3.iii.2020 from Tarmyiah/

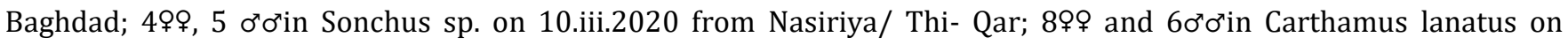
20.iii.2020 from Basra; 5ㅇ,5 ơ ơin Cucurbita pepo on 29.iii.2020 from Samarra.

Distribution: Iraq[15]; Turkey[33]; Portugal[20]; Norway, UK, Germany, Sweden, Netherlands, Finland, Pakistan, Korea, China, Japan, India, Portugal, South Africa, Eritrea, , Finland, Spain, Israel, Malta, Kenya, Ethiopia, Afghanistan, Zimbabwe, Italy, Denmark, Hungary and Austria[30].

\section{Genus, Japanagromyza Sasakawa, 1958}

\subsection{Japanagromyza salicifolii Collin, 1911}

Synonyms: Agromyza salicifolii Collin, 1911

Material examined: $39 \%$ specimens in poplar trees and Quercus L. (Fagaceae) on 4.iv.2020 from Mosul.

Distribution: Iraq [15] (Spencer, 1981); Palearctic Region [29]; Turkey [19]; Portugal [20].

Genus, Liriomyza Mik, 1894

Synonyms: Agrophila Lioy, 1864

Antineura Melander, 1913

Haplomyza Hendel, 1914

Praspedomyza Hendel, 1931

Triticomyza Blanchard, 1938

\subsection{Liriomyza brassicae (Riley, 1885)}

Synonyms: Liriomyza hawaiiensis Frick, 1952

Liriomyza ornephila Garg, 1971

Oscinis brassicae Riley, 1885 
Phytomyza mitis Curran, 1931

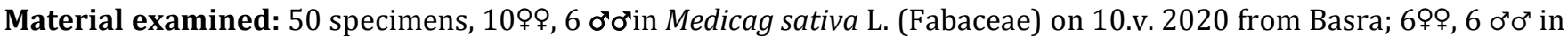
Pistum sativum on 17.iii.2020 from Kerbala; 10ㅇ, $10 \sigma^{\top} \sigma^{\prime}$ in Vigna mungo (L.) Hepper (Fabaceae) on $20 . i i$.2020 from Baghdad; 1 \%, $1 \sigma^{\top}$ in Dolichos sesuipedialis on 4.iv.2020 from Baghdad.

Distribution: Iraq [25]; Ethiopian, Australian, Nearctic. Neotropical and Palaearctic regions [29]; Turkey [19].

\subsection{Liriomyza bryoniae (Kaltenbach, 1858)}

Synonyms: Agromyza bryoniae Kaltenbach, 1858

Liriomyza citrulli Rohdendorf, 1950

Liriomyza hydrocotylae Hering, 1930

Liriomyza mercurialis Hering, 1932

Liriomyza nipponallia Sasakawa, 1961

Liriomyza solani Hering, 1927

Liriomyza triton Frey, 1945

Common name: Tomato Leaf-miner

Material examined: 40 specimens, 5ㅇ,5 ơ $\sigma^{\top}$ in Cucribta maxima Duchsne (Cucurbitaceae) on 21.iii.2020 from Kerbala;

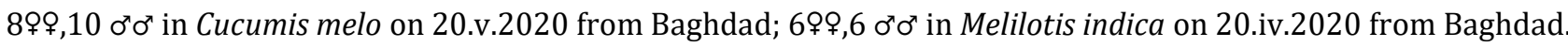

Distribution: Iraq [21]; Oriental and Palearctic Regions [29]; Turkey [19]; Portugal [20].

\subsection{Liriomyza congesta (Becker, 1903)}

Synonyms: Agromyza congesta Becker, 1903

Liriomyza centaureana Hering, 1936

Liriomyza leguminosarum Meijere, 1924

Liriomyza minima Hendel, 1931

Liriomyza nigripleura Ryden, 1956

Liriomyza parva Hendel, 1931

Liriomyza taraia Garg, 1971

Common name: Broad bean, bean and pea leaf-miner

Material examined: 20 specimens, 6९९, 6 ơ $^{\top}$ in Brassica rapa L. (Brassicaceae) on 20.ii.2020 from Baghdadsds; 499,4 $\sigma^{\top} \sigma^{\top}$ in Melilotis indica on 28.iv.2020 from Kerbela.

Distribution: Iraq [25]; Oriental and Palaearctic Regions [29]; Turkey [19]; Portugal [20].

\subsection{Liriomyza orbona (Meigen, 1830)}

Synonyms: Agromyza fuscolimbata Strobl, 1900

Agromyza orbona Meigen, 1830

Liriomyza fuscolimbata (Strobl, 1900)

Liriomyza orbonella Hendel, 1931

Material examined: previously recorded 19o' from south of Mosul on 13.iii.1979 [15].

Distribution: Iraq [15]; Palaearctic Region [29]; Turkey [19]; Portugal [20].

\subsection{Liriomyza sativae Blanchard, 1938}

Synonyms: Agromyza subpusilla Frost, 1943

Lemurimyza lycopersicae Pla \& de la Cruz, 1981

Liriomyza canomarginis Frick, 1952

Liriomyza guytona Freeman, 1958

Liriomyza minutiseta Frick, 1952

Liriomyza munda Frick, 1957

Liriomyza propepusilla Frost, 1954

Liriomyza properpusilla Frost, 1954

Liriomyza pullata Frick, 1952

Liriomyza verbenicola Hering, 1951

Common name: Vegetable leaf miner

Material examined: 30 specimens, 6\%९, 6 ơ ơin Melolitis indica (L.) All. (Fabaceae) on 25.iv.2020 from Baghdad; 10 $\%$, $8 \sigma^{\prime} \sigma^{\pi}$ in Cucurbita moschata Duchesne (Curcurbitaceae) on 20.x.2020 from Duhok, North of Iraq.

Distribution: Iraq [34]; Ethiopian, Australian, Nearctic. Neotropical and Palaearctic Regions [29]; Bangladesh [31]. 


\subsection{Liriomyza strigata Meigen, 1830}

\subsection{Liriomyza strigata Meigen, 1830}

Synonyms: Agromyza galeopsios Hardy, 1853

Agromyza pumila Meigen, 1830

Agromyza strigata Meigen, 1830

Agromyza violae Curtis, 1844

Liriomyza galeopsios (Hardy, 1853)

Liriomyza pumila (Meigen, 1830)

Liriomyza violae (Curtis, 1844)

Material examined: 20 specimens, 8ㅇ, $80^{\pi} \sigma^{\pi}$ in Brassica rapa L. (Brassicaceae) on 2.iv.2020 from Al-Nejef; $39 \%, 1 \sigma^{7}$ in Pisum sativum on 10.iv.2020.

Distribution: Iraq [21]; (Oriental and Palearctic Regions [29]; Turkey [19].

\subsection{Liriomyza trifolii Burgess, 1880}

Synonyms: Agromyza phaseolunata Frost, 194

Agromyza phaseolunulata Frost, 1943

Liriomyza alliovora Frick, 1955

Liriomyza phaseolunulata (Frost, 1943)

Oscinis trifolii Burgess, 188

Common name: American serpentine Leaf-miner

Material examined: 10 specimens, (6९९, $4 \sigma^{x} \sigma^{x}$ ) in Ricinus communis L. (Euphorbiaceae) on 28.x.2020 from Baghdad.

Distribution: Iraq [35]; Ethiopian, Australian, Nearctic, Neotropical and Palaearctic Regions [29]; Turkey [33].

Genus, Napomyza Westwood, 1840

Synonyms: Dinevra Lioy, 1864

Napomyia Schiner, 1868

\subsection{Napomyza lateralis (Fallen, 1823)}

Synonym: Phytomyza lateralis Fallén, 1823

Common name: Calendula fly

Material examined: 2specimens, $191 \sigma^{x}$ in Stachys sp. on 22.iii.2020 from Kirkuk.

Distribution: Iraq [15]; Nearctic and Palaearctic Regions [29]; Turkey [19]; Portugal [20].

Genus: Phytomyza Fallén, 1810

Synonyms: Lonicera Meijere, 1924

Phythomyza Rondani, 1874

Phytomyia Haliday, 1833

\subsection{Phytomyza ferulivora Griffiths, 1956}

Material examined: 10 specimens, (4\%९, 6 o $^{\top} \sigma^{7}$ ) in Daucus carota L. (Apiaceae) on 10.iii.2020 from Baghdad. Distribution: Iraq [15]; Palaearctic Region [29].

\subsection{Phytomyza ranunculi (Schrank, 1803)}

Synonyms: Musca ranunculi Schrank, 1803

Phytomyza albipes Meigen, 1830

Phytomyza cinereovittata Zetterstedt, 1848

Phytomyza cinerovittata Zetterstedt, 1848

Phytomyza citrina Roser, 1840

Phytomyza flava Fallen, 1823

Phytomyza flaveola Fallen, 1810

Phytomyza flavoscutellata Fallen, 1823

Phytomyza flavoscutellata Meigen, 1830

Phytomyza flavotibialis Strobl, 1902

Phytomyza incisa Macquart, 1835 
Phytomyza islandica Ryden, 1953

Phytomyza maculipes Brulle, 1833

Phytomyza maculipes Zetterstedt, 1848

Phytomyza pallida Meigen, 1830

Phytomyza pentalinearis Kuroda, 1954

Phytomyza praecox Meigen, 1830

Phytomyza ranunculi Kaltenbach, 1867

Phytomyza ranunculi Robineau-Desvoidy, 1851

Phytomyza scutellata Meigen, 1830

Phytomyza tenuipennis Singh \& Ipe, 1973

Material examined: 4 specimens, (2\%९,2 $\left.\sigma^{\top 7} \sigma^{\top}\right)$ in Anemon cornaria L. (Ranunculaceae) on 30.v.2020 from Baghdad.

Distribution: Iraq [15]; Nearctic, Oriental and Palaearctic Regions [29]; Turkey [19]; Portugal [20].

\subsection{Phytomyza rufipes Meigen, 1830}

Synonyms: Phytomyza bistrigata Strobl, 1906

Phytomyza brassicae Hardy, 1853

Phytomyza femoralis Brischke, 1881

Phytomyza genislatissimus Strobl, 1893

Phytomyza ruficornis Zetterstedt, 1848

Phytomyza sulphuripes Meigen, 1830

Phytomyza suphuripes Meigen, 1830

Common name: Cabbage leaf- miner

Material examined: 15 specimens, (8ㅇ, 7 ơ $\left.\sigma^{r}\right)$ in Brassicae rapa L. (Brassicaceae) on 28.ii.2020 from Basra.

Distribution: Iraq [15]; Nearctic, Neotropical and Palaearctic Regions [29]; Turkey [19]; Portugal [20].

\subsection{Phytomyza tetrasticha Hendel, 1927}

Material examined: 12 specimens, (8\%९, $\left.4 \sigma^{\top} \sigma^{r}\right)$ in Mentha longifolia (L.) L. (Lamiaceae) on 20.iii.2020 from Baghdad. Distribution: Iraq [15]; Palaearctic Region [29]; Turkey [19]; Portugal [20].

Family: Anthomyiidae

Genus, Pegomya Robineau-Desvoidy, 1830

Synonyms: Pegomaya Macquart, 1835

Patermia Albuquerque, 1954

Forai Robineau-Desvoidey, 1830

Forie Macquart, 1835

\subsection{Pegomya bicolor Wiedemann, 1817}

Synonyms: Anthomyia mitis Meigen, 1826

Anthomyia rumicis Bouché, 1834

Pegomya cinereorufa (Ringdahl, 1930)

Pegomya mitis (Meigen, 1826)

Pegomyia cinereorufa Ringdahl, 1930

Pegomyia sapporensis Kato, 1941

Zabia longipes Robineau-Desvoidy, 1830

Material examined: 4specimens, 2\%, ơ ơ 2 in Rumex sanguineus L. (Polygonaceae) on 25.iv.2020 from Baghdad.

Distribution: Iraq [36]; Japan[37]; Czech Republic[38]; India[39]; Finland[40]; Scotland[41]; Iran[42]; UK [43]; Palaearctic [44]; Korea[11].

\subsection{Pegomya cunicularia (Rondani, 1886)}

Synonyms: Chorthophila cunicularia Rondani, 1866

Chortophila cunicularia Rondani, 1866

Pegomya mixta Villeneuve, 1922

Pegomyia tristriata Stein, 1908

Material examined: 5specimens, 3\%९, ơ ơ 2 in Chenopodium murale L. (Amaranthaceae) on 14.iv.2020 from Baghdad.

Distribution: Iraq [36]; Canary Island [45]; Spin [46]; Korea [11]. 


\subsection{Pegomya hyoscyami (Panzer, 1809)}

Synonyms: Anthomyia hyoscyami Panzer, 1809

Chortophila stlemba Giglio-Tos, 1893

Material examined: 4specimens, $2 \%$, ơ ơ2 in Chenopodium sp. L. (Amaranthaceae) in ix.2020.from Baghdad.

Distribution: Iraq[47]; Cyprus, Jordan, Israel, Egypt, Turkey, Libya, Europe[48]; Finland[40] Scotland[41]; Turkey[49];

Iran[42]; Korea[11]; Russia[50].

\subsection{Pegomya terebrans(Rondani, 1866)}

Synonyms: Pegomya sylvatica (Rondani, 1866)

Chorthophila brasiliensis Rondani, 1866

Pegomya incisa Villeneuve, 1923

Pegomyia unicolor Strobl, 1909

Material examined: 4 specimens, $2 \% \%$, and $\sigma^{\top} \sigma^{2} 2$ in Carduus nutans subsp. Platypus (Lang) Greuter (Asteraceae) on 18.1.2020 from Baghdad.

Distribution: Iraq [36]; Spin [46].

\section{Family: Drosophylidae}

Genus, Scaptomyza Hady, 1850

Synonyms: Alloscaptomyza Hackman, 1962

Boninoscaptomyza Okada, 1972

Bunostoma Malloch, 1932

Ctenoscaptomyza Frey, 1954

Dentiscaptomyza Hardy, 1965

Euscaptomyza Séguy, 1938

Exalloscaptomyza Hardy, 1965

Lauxanomyza Tsacas \& Cogan, 1976

Macroscaptomyza Frey, 1954

Metascaptomyza Hackman, 1959

Rosenwaldia Malloch, 1934

Scaptomyzella Hendel, 1928

Scaptomyzetta Hendel, 1928

Tantalia Malloch, 1938

Tristanomyia Frey, 1954

Trogloscaptomyza Frey, 1954

\subsection{Scaptomyza flava (Fallen, 1823)}

Synonyms: Drosophila flava Fallén, 1823

Notiphila flaveola Meigen, 1830

Scaptomyza apiralis Duda, 1921

Scaptomyza flaveola (Meigen, 1830)

Material examined: 20 Specimens, $5 \%$ $9 \sigma^{\top} \sigma^{\top}$ in Raphanus raphanistrum subsp. sativus (L.) Domin (Brassicaceae) on 20.iii.2020 from Basra and 6ㅇ, $40^{\top} \sigma^{\top}$ on 30.iii.2020. From Baghdad.

Distribution: Iraq [51]; New Zealand [52]; Fennoscandia and Denmark [53]; UAE [54] Hawaii [55]; Malta [56]; Arizona [57]; UK [58].

\section{Order: Lepidoptera}

Family: Gelechiidae

Genus, Tuta Kieffer \& Jörgensen, 1910

\subsection{Tuta bsoluta (Meyrick, 1917)}

Synonyms: Phthorimaea Absolute Meyrick, 1917

Gnorimoschema absoluta (Meyrick, 1917)

Common names: Tomato leafminer and tomato pinworm. 


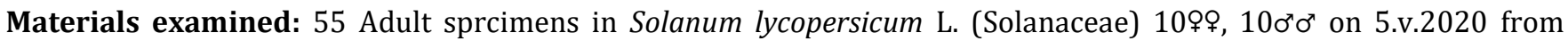

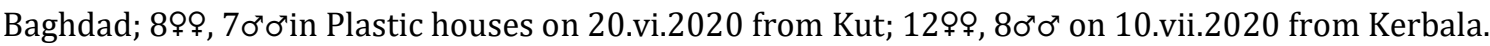

Distribution: Iraq [59] Argentina, Brazil, Paraguay, Venezuela [60]; Spain [61]; European and North African [62].

\section{Family, Gracillariidae}

Genus, Phyllocnistis Zeller, 1848

\subsection{Phyllocnistis citrella Stainton, 1856}

Synonyms: Phyllocnistis citricola (Shiraki, 1913)

Phyllocnistis minutella van Deventer, 1904

Common name: Citrus Leaf Minor (CLM)

Material examined: Many leaves of mandarin orange Citrus reticulata Blanco (Rutaceae) were infested by CLM on 22.iii.2020 from Baghdad; and Citrus L. (Rutaceae) infested leaves collected on 10.ii.2020 from Kerbala.

Distribution: Iraq[63]; Egypt[64]; Morocco[65]; Syria[66]; Pakistan[67]; Turkey[68]; Greece[69]; Algeria[70].

\section{Conclusion}

The investigation showed the agromyzid flies the most important leaf miners attacked many various host plant types and caused damages The research noticed that the insects were found in the leaves of Fabaceae plants more than other Families.

\section{Compliance with ethical standards}

\section{Acknowledgments}

The authors are grateful to the farmers who help us when collecting the infesting leaves plants from their farms in different localities in Iraq.

\section{Disclosure of conflict of interest}

The authors have no conflicts of interest to declare.

\section{References}

[1] http: //www.ukflymines.co.uk/index.php available 8.12.2021.

[2] Spencer KA. Diptera: Agromyzidae. Handbooks for the identification of British insects, R. Entomol. Soc. London. 1972; 10(5): 136.

[3] Spencer KA. A synopsis of the Oriental Agromyzidae (Diptera). Trans. R. Entomol. Soc. Lond. 1961; 113(4): 55100.

[4] Spencer KA. A synopsis of the Neotropical Agromyzidae (Diptera). Trans. R. Entomol. Soc. Lond. 1963; 115(12): 291-389.

[5] Hennig W. Die Familien der dipteral Scizophora und ihre phylogenetischen verwandschafstsbeziehug. Beiträge zur Entomologie. 1958; 8: 505-688.

[6] Spencer KA. Agromyzidae. In (McAlpine J. F. Ed.) Manual of Nearctic Diptera volume 5 Research. Branch Agriculture Canada, Monoghaph. 1987; 28. 675-1332.

[7] Oldroyd H. Diptera, Introduction and key to families. Handbk. Identif. Br. Insects, R.Entomol. Soc. Lond. 1970; 9(1): 104.

[8] Unwin DM. A key to the families of British Diptera. Field studies. 1981; 5: 513-553.

[9] Scudder G GE, Cannings R. A. The Diptera families of British Colombia. The Diptera families of British Colombia. 2006; 1-158.

[10] Suh SJ. Taxonomic investigation of the family Anthomyiidae from Korea. M.Agr. thesis, Kyungpook Nat. Univ. $1985 ; 39$. 
[11] Suh SJ. Anthomyiidae, Arthropoda: Insecta: Diptera: Muscoidea. Insect Fauna of Korea. 2019; 5(4): 1-227.

[12] Brake I. Bachli GE. World Catalogue of Insect: Drosophilidae (Diptera). Stenstrup, Denmark. 2008 ; $9: 412$.

[13] Bock IR. Drosophilidae of Australia. II. Scaptomyza (Insecta: Diptera). Aust. J. Zool. 1977; 25: $337-345$.

[14] Muszinski T, Lavendowski IM, Maschio LMA. 'Constatação de Scrobipalpula absoluta (Meyrick) (Lepidoptera: Gelechiidae), como praga do tomateiro (Lycopersicon esculentum Mill.), no litoral do Paraná', An. Soc. Entomol. Brasil. 11: 291-292.

[15] Spencer KA. Agromyzidae (Diptera) in Iraq. Entomologist's Gazentte. 1981; 32: 177-180.

[16] Spencer KA. The Agromyzidae (Diptera) of Fennoscandia and Denmark. Fauna entomologica scandinaviaca. 1976; 5(1): 1-304; 5(2): 305-606.

[17] Spencer KA. Host specialization in the world Agromyzidae (Diptera). Series Entomologica, Kluwer Academic Publishers, Dordrecht. 1990; 45: 444.

[18] Scheirs J, Bruyn L De, Verdyck P. Leafminers (Diptera; Agromyzidae) of the Belgian fauna. Bulletin et Annales de la Société royale belge d'Entomologie. 1993; 129: 113-119.

[19] Cİvelek HS, Cikman E, Dursun O. Revised checklist of Turkish Agromyzidae (Diptera) fauna of Turkey. Turkish Journal of Zoology. 2009; 33: 349-358.

[20] Černý M, Andrade R, Gonçalves A R, von Tschirnhaus M. New records of Agromyzidae (Diptera) from Portugal, with an updated checklist. Acta Musei Silesiae, Scientiae Naturales. 2018; 67: 7-57.

[21] Al Ali AS. Phytophagous and entomophagous insects and mites of Iraq. Nat. Hist Res. Center, $1977 ; 33: 142$.

[22] Černý M, Merz B. 2006. New records of Agromyzidae (Diptera) from Palaearctic Region. Mitteilungen der schweizerischen entomologischen Gesellschaft. 2006; 79: 77-106.

[23] Černý MNew faunistic data on the Agromyzidae (Diptera) from the West Palaearctic Region. Klapalekiana. 2009; 45: 9-21.

[24] Černý M, von Tschirnhaus M, Winqvist K. First records of Palaearctic Agromyzidae (Diptera) from 40 countries and major islands. Acta Musei Silesiae, Scientiae Naturales. 2020; 69: 193-229.

[25] Al- Azawi AF Agromyzid Leaf miners and their parasitites in Iraq. Bull. Entomol. Res. 1967; 57(2): $285-287$.

[26] Bei-Bienko GY, Steyskal GC. Keys to the Insects of the European Part of the USSR, Diptera and Siphonaptera, Amerind Publishing Co., New Delhi. 1988; 5(11).

[27] GBIF Secretariat. GBIF Backbone Taxonomy. Checklist dataset https: //doi.org/10.15468/39omei accessed via GBIF.org on. 2021-11-15.

[28] Benavent- Corai, J, Martinez M, Jimenez Peydro R. Catalogue of the host -plants of the world Agromyzidae(Diptera), Part 1: List of Agromyzidae species and their hosts-plants, Part2: List of hosts-plants and Agromyzidae associated. Bollettino Di Zoologia Di Bachicoltura, Ser. II, 2005; 37: 1-50.

[29] Benavent- Corai J, Martinez M, Jimenez Peydro R. Catalogue of the host -plants of the world Agromyzidae(Diptera), Part 1: List of Agromyzidae species and their hosts-plants, Part2: List of hosts-plants and Agromyzidae associated. Bollettino Di Zoologia Di Bachicoltura. 2005; Ser. II, 37: 1-50.

[30] GBIF Secretariat. GBIF Backbone Taxonomy. Checklist dataset https: //doi.org/10.15468/39omei accessed via GBIF.org on. 2021-11-17.

[31] Mazumdar S, Bhuiya BA. Vegetable leafminers (Diptera: Agromyzidae) and their plant hosts in Bangladesh. Journa of Threatened Taxa. 2014; 6(6): 5894-5899.

[32] Radjabi Gh, Hosseyni SM, Mansoor-Ghaazi M. Diptera species occurring in wheat and barley in Iran. Applied Entomology and Phytopathology. 1997; 64(1, 2): 60-71.

[33] Bayhan SÖ, Kaplan M, Bayhan E. Agromyzid (Diptera) species and their parasitoids in Batman Province, Turkey. Middle East Journal of Sciense. 2017; 3(2): 98-106.

[34] Abdul Rassoul MS, Al-Saffar HH. Survey of the genus Liriomyza Mik. (Diptera: Agromyzidae) of Iraq. Advances in Bioresearch. 2013; 4(3): 92-94.

[35] Al-Jorany RS, Flaih SK, Alhamawandy Sh A, Al-Saffar HH. New record of Liriomyza trifolii (Burgess, 1880) (Diptera; Agromyzidae) in Baghdad, Iraq. Int. j. Curr. Microbiol. Sci. 2016; 5(5): 205-211. 
[36] Mekhlif AF. Records of some leaf miner of Anthomyiidae, (Diptera) and their host plants in Iraq. Bulletin of Iraq Natural History Museum. 1999; 1(9): 117-121.

[37] Suwa M. Anthomyiidae of Japan (Diptera). Insecta Matsumurana New Series. 1974; 4: 247.

[38] Komzáková1 O, Barták M, Bartáková D, Kubík Š. Community structure of Anthomyiidae (Diptera) of six peat-bogs in the Šumava Mts (Czech Republic). Biologia. 2011; 66/3: 518-527, Section Zoology.

[39] Suwa M. Some Anthomyiidae from India (Diptera). Insecta Matsumurana. 1981; 22: 15-28.

[40] Fischer M, Koponen M. A survey of Opiinae (Hymenoptera, Braconidae) of Finland, part 2. - Entomol. Fennica. 1999; 10: 129-160.

[41] Skidmore P. A review of the Diptera of the Western Isles of Scotland. Dipterists Digest. 2008; 15(2): 99-194.

[42] Gadallah NS, Ghahari H, Peris-Felipo FJ, Fischerm M. Updated checklist of Iranian Opiinae (Hymenoptera: Braconidae). Zootaxa. 2016; 4066(1): 001-040.

[43] Telfer MG. Invertebrate survey of Tilbury2. Report to Bioscan (UK). 2017; 142.

[44] Tschorsnig, H-P. Preliminary host catalogue of Palaearctic Tachinidae (Diptera). 2017.

[45] First version, provided online on http: //www.nadsdiptera.org/Tach/WorldTachs/CatPalHosts/Home.html.

[46] Michelsen. V, Briez M. The Anthomyiidae (Diptera) of the Canary Island. Ent. Scan. 1985; 16: 777-304.

[47] Suwa M, Blasco-Zumeta J. Some Anthomyiid flies from Monegros, Spain (Diptera: Anthomyiidae). Insecta Matsumurana. 2003; 60: 43-54.

[48] Hussain AA. Provisional list of Insect pests and Bibliography of Insect fauna of Iraq. Bulletin of the College of Science Baghdad. 1963; 7: 43 - 83.

[49] Gentry JW. Crop Insects of Northeast Africa-Southwest Asia. Agriculture Handbook. 1965; $273: 214$.

[50] Beyarslan A. A faunal study of the subfamily Doryctinae in Turkey (Hymenoptera: Braconidae). Turkish Journal of Zoology. 2015; 39: 126-143.

[51] Belokobylskij SA, Lelej AS. Annotated catalogue of the Hymenoptera of Russia. Volume II, Apocrita: Parasitica, Supplement № 8, 596 Proceedings of the Zoological Institute of the Russian Academy of Sciences. 2019.

[52] Mehdi HA, Najim ShA, Aufi BG. Chemical and biological control of radish leave miner insect Scaptomyza flava Fallen (Diptera: Drosophilidae) under the Conditions of Basrah Province. Syrian Journal of Agricultural Research - SJAR. 2019; 6(4): 549-556.

[53] Seraj AA. Biology and host plant relationships of Scaptomyza flava leaf miner. A thesis of degree Doctor of Philosophy in Entomology, Plant Science Department, Massey University, Palmerstone North New Zealand. 1995.

[54] Bachli G, Vilela CR, Escher SA, Saura A. The Drosophilidae (Diptera) of Fennoscandia and Denmark. Fauna Entomologica Scandinavica. 2004; 39: 1-187.

[55] Sidorenko VS, Nakonechnaya OV. Order Diptera, Family Drosophilidae. Arthropod Fauna of the UAE. 2010; 3: 661-672.

[56] Lapoint, RT, O'Grady PM, Whiteman NK. Diversification and dispersal of the Hawaiian Drosophilidae: the evolution of Scaptomyza. Molecular phylogenetics and evolution. 2013; 69(1): 95- 108.

[57] Ebejer MJ. A short note on additional records of fruitfly (Diptera, Drosophilidae) from Malta. Bulletin of Entomological Society of Malta. 2015; 7: 143.

[58] Goldman-Huertasa B, Robert RF, Lapointa RT, Faucherb CP, Hildebrandb JG, Whitemana NK. Evolution of herbivory in Drosophilidae linked to loss of behaviors, antennal responses, odorant receptors, and ancestral diet. Pans. 2015; 112 (10): 3026-3031.

[59] Hackett T, Sauve AMC, Davies NE, Montoya D, Tylianakis J, Memmott J. Reshaping our understanding of species'roles in landscape-scale networks. Ecology Letters. 2019.

[60] Abdul Razzak AS, Al-Yasiri I I, Fadhil HQ. First record of tomato borer (tomato moth) Tuta absoluta (Meyrick) (Lepidoptera: Gelechiidae) on tomato crop in Iraq. Arab and Near East Plant Protection Newsletter. 2010; 51 : 31.

[61] Urbaneja A, Vercher R, Navarro V, Garcı'a Marı' F, Porcuna JL. La polilladeltomate, Tuta absoluta. Phytoma Espana. 2007; 194: 16-23. 
[62] Desneux N, Wajnberg E, Wyckhuys KAG, Burgio G, Arpaia SCA, , Narva'ezVasquez S C A, lez-Cabrera J G, Ruescas D C, Tabone E, Frandon J, Pizzol, J, Poncet C, Cabello T, Urbaneja A. Biological invasion of European tomato crops by Tuta absoluta: ecology, geographic expansion and prospects for biological control. Journal Pest Science. 2010; 83: $197-215$.

[63] Gentry JW. Crop Insects of Northeast Africa- Southwest Asia. Agricultural Research Service, United States Department of Agriculture, Agriculture Handbook 1965; 273, Washington, 210.

[64] Abd El-Aziz SE. Biological studies of citrus leaf miner, Phyllocnistis citrella (Staint.) in Egy Bulletin of Entomological Society of Egypt. 1995; 73: 97-105.

[65] Boughdad A, Bouazzaoui Y, Abdelkhalek L. Pest status and biology of citrus leaf miner, Phyllocnistis citrella in Morocco. Proceedings of $5^{\text {th }}$ International Conference on Pest in Agriculture. 1999; 2: 251-259.

[66] Abo-Kaf M, Aslan A, Ahmed I. Morphology and biology of citrus leaf miner Phyllocnistis citrella (Lepidoptera: Gracillaridae) in Syria. Arab Journal of Plant Protection. 2006; 24(1): 45-48.

[67] Mustafa I, Raza AM, Aqeel MA, Ahmed H, RiazKhan M, Ahmed I, Arshad M. Correlation of Citrus Leaf Miner (Phyllocnistis citrella Stainton) with Snail Population in District Sargodha, Punjab, Pakistan. Pakistan Journal of Zoology. 2013; 45(2): 453-458.

[68] Elekcioğlu NZ, Uygun N. Population Fluctuation of Citrus Leafminer, Phyllocnistis citrella Stainton (Lepidoptera: Gracillariidae) and its Parasitoids in the Eastern Mediterranean Region of Turkey. Pakistan J. Zool. 2013; 45(5): 1393-1403.

[69] Tsagkarakis AH, Kalaitzaki AP, Lykouressis DP. Phyllocnistis citrella and its parasitoids in three citrus species in Greece. Phytoparasitica. 2013; 41: 23-29.

[70] Dahmane M, Chakali G. Distribution pattern of developmental stages of Phyllocnistis citrella Stainton (Lepidoptera: Gracillariidae) on the surface of citrus leaves. Polish Journal of Entomology. 2020; 89(1): 1-6. 